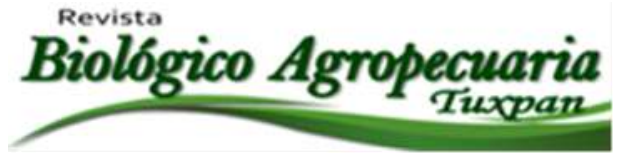

\title{
Proyecto de inversión para una microprocesadora de lácteos en la localidad de Naica, municipio de Saucillo, Chihuahua.
}

Investment project for a microbusiness of dairy in the town of Naica, municipality of Saucillo, Chihuahua.

\author{
Magaña Magaña José ${ }^{1 凶}$ Eduardo, Villarreal Ramírez Víctor Hugo ${ }^{1}$, Kiessling Davison Christian Mauricio ${ }^{1}$ \\ Terrazas Gómez Marina Imelda ${ }^{1}$ y González Domínguez Janeth Guadalupe ${ }^{1}$ \\ ${ }^{1}$ Facultad de Ciencias Agrícolas y Forestales-Universidad Autónoma de Chihuahua, responsable de la \\ publicación. \\ Autor para correspondencia.
}

Recibido: 15/07/2017

Aceptado: 10/12/2017

\section{RESUMEN}

La presente investigación tiene un enfoque mixto. Se realizó un proyecto de inversión para una microprocesadora de lácteos en la localidad de Naica, municipio de Saucillo, Chihuahua. Se aplicó un cuestionario a 91 habitantes de la localidad para conocer la tendencia de consumo de productos lácteos en dicha región. Se realizó un panel de expertos en procesamiento de quesos y se identificó la materia prima, costos y cantidad de producto que se requiere para su elaboración. Se realizó un análisis estadístico referente a las tendencias de consumo y precios que estarían dispuestos a pagar por los productos lácteos que la empresa ofertara. El estudio de mercado arrojo información de la factibilidad de ventas de queso asadero, cheddar, chopitos y jamoncillo. El proyecto de inversión siguió el formato de SAGARPAFAPPA, con un horizonte de proyecto de cinco años (2017- 2022). Se calculó la Tasa Interna de Retorno (TIR), siendo esta del $28 \%$ y considerando la tasa de actualización del $15 \%$, el proyecto recupera los $\$ 537,561.24$ de inversión inicial y es factible financieramente. Además se obtuvo el Valor Actual Neto (VAN 15\%) de $\$ 155,390.20$, que evaluándolo con el criterio de aceptar el proyecto con una VAN mayor o igual a cero, el proyecto es económicamente viable.

Palabras clave: Proyecto, Inversión, Microprocesadora, Lácteos, Queso.

\begin{abstract}
ABSTRAC
This research has a quantitative and qualitative approach. It is an investment for a microprocessor dairy project in the town of Naica, municipality of Saucillo, Chihuahua. A questionnaire was administered to 91 residents of the town to meet the trend of consumption of dairy products in the region. Besides was conducted a panel of experts in which were identified process of cheese, cost and the quantity of product required for its development. A statistical analysis was realized for knowing trends in consumption and prices that the potential consumers were able to pay for dairy products that the company established. The
\end{abstract}


market research presented information that is feasibility to produce sell asadero cheese, cheddar, chopitos and jamoncillo. The investment project followed the format of SAGARPA-FAPPA, with a project horizon of five years (2017-2022). An internal rate of return (IRR) was calculated, which was $28 \%$, and considering the update rate of $15 \%$, the project is going able to recuperate the 537,561.24 Mexican pesos of initial investment therefore is financially feasible. Also was obtained the net present value (VAN $15 \%$ ) which was $155,390.20$ Mexican pesos, using the criteria of accepting each project with a range greater than or equal to zero, this project is economically viable.

Keywords: Investment, Project, Microprocessor, Dairy, Cheese.

\section{INTRODUCCIÓN}

En la presente investigación se muestran los elementos básicos para la elaboración de un proyecto de inversión para una microprocesadora, en donde se contempla la fabricación de productos lácteos en la localidad de Naica, Municipio de Saucillo, Chihuahua. Es preciso referir que el Gobierno Federal cuenta con apoyos económicos para aquellas personas que tengan pensado poner en marcha un pequeño negocio. Uno de esos apoyos es el programa FAPPA, el cual trata de apoyar aquellas personas físicas o morales a realizar un proyecto productivo, preferentemente que habiten en Núcleo Agrario con infraestructura básica, equipo de insumos, especies pecuarias y asesoría técnicas para la implementación de proyectos productivos. También el FAPPA apoya a grupos sin formalidad jurídica y a grupos legalmente constituidos. (SAGARPA, 2017)

La localidad de Naica está situada en el Municipio de Saucillo (en el Estado de Chihuahua). Hay 4938 habitantes. Naica está a 1328 metros de altitud. En la localidad hay 2492 hombres y 2446 mujeres. $\mathrm{El}$ ratio mujeres/hombres es de 0,982, y el índice de fecundidad es de 2,56 hijos por mujer. Del total de la población, el 13,22\% proviene de fuera del Estado de Chihuahua. El 1,54\% de la población es analfabeta (el 1,40\% de los hombres y el $1,68 \%$ de las mujeres). El grado de escolaridad es del 8.52 (8.62 en hombres y 8.43 en mujeres). El 0,65\% de la población es indígena, y el 0,28\% de los habitantes habla una lengua indígena. El $0,00 \%$ de la población habla una lengua indígena y no habla español. El 31,00\% de la población mayor de 12 años está ocupada laboralmente (el $49,00 \%$ de los hombres y el $12,67 \%$ de las mujeres). (Pueblos América, s.f.)

Por otra parte, en el 2015 afecto el cierre de la mina ya que muchos habitantes se quedaron sin ingresos, también hubo desabastecimiento de agua, aumentaron las pérdidas de cultivos y animales. El desempleo, el alcoholismo, la drogadicción y la violencia se incrementaron a raíz del cierre de la mina en Naica, lo que dejó sin sustento a 460 familias y ocasionó un éxodo de varones en busca de un mejor porvenir para sus familias. (Rivas, 2017)

La localidad de Naica está incorporada en actividades agropecuarias, pecuarias y mineras, acciones que fortalecen la formación de los proyectos productivos, es por ello que la presente investigación se torna relevante, dado que el producto final del estudio es la realización de un proyecto de inversión para una microprocesadora de lácteos, en donde los beneficiarios principales serán los habitantes de dicha localidad, dado que se pretende conocer la dinámica económica de esa región para la generación de empleos a nivel micro. 
El objetivo es contribuir al desarrollo económico del ejido Naica a través de elaborar un proyecto de inversión de productos lácteos.

\section{MATERIALES Y MÉTODOS}

A. Tipo y diseño de la investigación. Con el fin de dar respuesta al objetivo general y a los específicos, se realizó un estudio de campo, transaccional y relacional por que presenta un panorama de los elementos que intervienen en la elaboración de un proyecto de inversión. Se aplicó un cuestionario a 91 habitantes de la localidad de Naica. Se les aplicó dicho instrumento a conveniencia, esto es, que no se hizo un ejercicio de muestreo aleatorioprobabilístico para obtener la muestra poblacional. Las técnicas auxiliares empleadas para la elaboración del presente estudio fueron: Word, Excel y el software estadístico SPSS.

B. Nivel de la Investigación. El presente trabajo se encuentra en un nivel descriptivo, ya que simplemente se observan los fenómenos tal y como se dan en su contexto natural, para después analizarlos, sin emplearse estímulos o condicionantes para manipular las variables. (Hernández, Fernández, \& Baptista, 2004).

C. Alcance de la investigación. Es de tipo no experimental con el propósito de entender el fenómeno tal como se presenta.

D. Naturaleza de la investigación. La presente investigación es de naturaleza mixta (cuantitativa y cualitativa). Es cuantitativa ya que se analizaron datos estadísticos y se torna cualitativa debido a que se entrevistaron a los actores principales de la investigación, ejidatarios y microempresarios.

A continuación les presentar la maquinaria y equipo para el proceso de los quesos y la pasteurización, en donde el precio unitario del equipo y/o maquinaria se obtuvo del sitio electrónico (mercado libre, 2017).

Tanque de almacenamiento 500 a 1000 litros

Tanque de pasteurización

Tanque de enfriamiento

Tina de enfriamiento y batido

Cámara de refrigeración

Descremadora 80 litros por hora

Montacargas manual

\section{RESULTADOS}

Los resultados son el sustento para la propuesta planteada en el presente trabajo de investigación.

La mayoría de los ejidatarios encuestados en la localidad de Naica tienen 65 años o más, cabe señalar que el promedio de edad de los ejidatarios encuestados fue de 67 años.

Más de la mitad de los encuestados sí han consumido productos lácteos. El 64 por ciento de los encuestados sí les gustaría que se vendiera productos lácteos de buena calidad en el municipio de Saucillo; La mitad de las personas encuestadas no están interesadas en comprar jamoncillos, ver cuadro El 74 por ciento de los encuestados les interesa comprar queso; El 51 por ciento de los encuestados refirió que sí compraba dicho producto, mientras que 49 por ciento opinó lo contrario; Los chopitos es el producto de mayor preferencia de las personas encuestadas, ya que 66 por ciento respondió que sí les interesa comprar tal producto.

La mayoría de las personas encuestadas no saben cuánto pagar por adquirir un jamoncillo, sin embargo, 31 por ciento dijeron que están dispuestos a pagar 5 pesos por dicho producto; Las personas pagarán alrededor de 10 pesos por tal producto; Las personas encuestadas no tienen 
idea de cuánto pagar por una pieza de asadero, sin embargo, los que sí saben manifestaron que están dispuestos a pagar 12 pesos por dicho producto; Las personas estarían dispuestas a pagar por adquirir un chopito, siendo 8 pesos el precio que pagaría la mayoría por dicho producto; A las personas les interesa comprar productos lácteos los fines de semana con una tendencia de consumo de dos días por semana.

\section{CONCLUSIÓN}

De los productos lácteos contemplados para ofertar en la presente propuesta de proyecto de inversión, se tiene que de los 4 productos (jamoncillo, asadero, queso tipo chihuahua y chopito de queso fresco), el asadero se tiene contemplado que del 100 por ciento del total de las ganancias, es el que da la mayor parte de la misma con 45.4 por ciento, seguido del queso tipo chihuahua con 26.4 por ciento, sumando entre ambos el 71.8 por ciento.

\section{LITERATURA CITADA}

Bello, J. M., Lizeldi, B. V., González, E. V., Manzo, A. S., Nochebuena, X. P., Quiñónez, E. I., \& Vázquez, C. S. (10 de Agosto de 2004). 5(7). Recuperado el 15 de Marzo de 2017, de http://www.revista.unam.mx/vol.6/num9/art 89/sep_art89.pdf

Carrasquillo, L. (26 de Junio de 2011). Recuperado el 18 de mayo de 2017, de https://incubadorademicroempresas.com/luc y/blog/definamos-que-es-una-microempresa

CreceNegocios. (s.f.). Obtenido de https://www.crecenegocios.com/lasrelaciones-publicas/

Directorio Estadístico Nacional de Unidades Económicas. (2017). Recuperado el Septiembre de 2017, de http://www.beta.inegi.org.mx/app/mapa/den ue/\#

eco-finanzas. (s.f.). Recuperado el Octubre de 2017, de http://www.ecofinanzas.com/diccionario/I/INVERSION.ht $\mathrm{m}$

Fernández, E. S. (2007). Los proyectos de inversión. Costa Rica: Tecnológica de Costa Rica.
Gestiopolis. (s.f.). Recuperado el 18 de Mayo de 2017 , de https://www.gestiopolis.com/componentesde-un-proyecto-de-inversion-presentacion/

Hernández, S. R., Fernández, C. C., \& Baptista, L. P. (2004). Metodología de la investigación. México: Macgrawhill.

Hernangómez, B. J. (s.f.). La empresa como organización: una propuesta de delimitación de su concepto. 226-237.

Llanos, M. (s.f.). Proyectos de inversión. Recuperado el Octubre de 2017, de https://proyectosinversion.wordpress.com/p royectos-de-inversion/

mercado libre. (2017). Recuperado el Agosto de 2017, de https://www.mercadolibre.com/

OBS Business School. (s.f.). Project Management. Recuperado el Octubre de 2017, de https://www.obs-edu.com/int/blog-projectmanagement/conceptos-fundamentalesdireccion-de-proyectos/que-es-un-proyectoelementos-fases-y-tipos

Pérez, P. j., \& Merino, M. (2009). Definición.DE. Recuperado el Octubre de 2017, de https://definicion.de/inversion/

Pueblos América. (s.f.). Recuperado el Septiembre de 2017, de https://mexico.pueblosamerica.com/i/naica/

Rivas, V. (11 de Febrero de 2017). El Heraldo de Chihuahua. Recuperado el Septiembre de 2017.

SAGARPA. (20 de Enero de 2017). Recuperado el 23 de Septiembre de 2017, de https://www.gob.mx/sagarpa/acciones-yprogramas/componente-proyectosproductivos-fappa

Secretaria de Economía. (2009). Acuerdo por el que se establece la estratificación de las micro, pequeñas y medianas empresas.

Secretaría de Economía. (marzo de 2012). Análisis del Sector Lácteo en México. Recuperado el 19 de Mayo de 2017, de http://www.economia.gob.mx/files/comunid ad_negocios/industria_comercio/informacio nSectorial/analisis_sector_lacteo.pdf

Thompson, B. J. (mayo de 2006). Promonegocios.net. Recuperado el 18 de Mayo de 2017, de https://www.promonegocios.net/proyecto/con cepto-proyecto.html 
Copyright (c) 2017 José E duardo Magaña Magaña, Victor Hugo Villarreal Ramirez, Chri stian Mauricio Kies sling Davison,

Marina Imel da Terrazas Gómez y Janeth Guadalupe González Dominguez

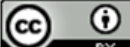

Este tex to está protegido por una licencia licencia Creative Commons 4.0

Usted es libre para Compartir —copiar y redistribuir el material en cualquier medio o form ato- y Adaptar el documento -remezdar, transformar y crear a partir del material- para cualquier propósito,, incluso para fines comerciales, siempre que cumpla la condición de:

Atribución: Usted debe dar crédito a la obra original de manera adecuada, proporcionar un enlace a la licencia, e in đicar si se han realizado cam bios. Puede hacerlo en cualquier form a razonable, pero no de form a tal que sugiera que tiene el apoyo del licenciante o 10 recibe por el uso que hace de la obra.

Resumendelicencia - Textocompletodelalicencia 